\title{
Germanica
}

\section{Laurence Guillon, Heidi Knörzer (Hrsg.) : Berlin und die Juden. Geschichte einer Wahlverwandschaft?}

Berlin, Neofelis Verlag, 2015, 212 Seiten.

\section{François Prolongeau}

\section{OpenEdition}

\section{Journals}

Édition électronique

URL : http://journals.openedition.org/germanica/3124

DOI : 10.4000/germanica.3124

ISSN : 2107-0784

\section{Éditeur}

Université de Lille

Édition imprimée

Date de publication : 30 décembre 2015

Pagination : 207-211

ISBN : 9782913857360

ISSN : 0984-2632

\section{Référence électronique}

François Prolongeau, « Laurence Guillon, Heidi Knörzer (Hrsg.) : Berlin und die Juden. Geschichte einer Wahlverwandschaft? », Germanica [En ligne], 57 | 2015, mis en ligne le 25 janvier 2016, consulté le 06 octobre 2020. URL : http://journals.openedition.org/germanica/3124 ; DOI : https://doi.org/10.4000/ germanica.3124 


\section{Comptes rendus de lecture}

Laurence Guillon, Heidi Knörzer (Hrsg.) : Berlin und die Juden. Geschichte einer Wahlverwandschaft? Berlin, Neofelis Verlag, 2015, 212 Seiten.

L'ouvrage coédité par Laurence Guillon et Heidi Knörzer constitue le neuvième volume d'une collection prévue de 11 volumes consacrée à l'histoire culturelle des Juifs à l'époque moderne (Jüdische Kulturgeschichte in der Moderne). Le présent ouvrage est constitué de contributions de chercheurs français et allemands. Après avoir donné dans l'introduction quelques repères sur l'histoire de la présence juive à Berlin, les éditrices reviennent sur le débat autour du concept de «symbiose judéo-allemande » qui opposa différents intellectuels juifs allemands dans l'entre-deux-guerres, pour finir par proposer l'idée d'une « affinité élective » (Wahlverwandschaft), pour qualifier le rapport des Juifs à la capitale allemande.

Le livre se divise en deux grandes parties (« topographies de la grande ville » et «vie intellectuelle et musique ») réunissant chacune quatre articles, que précède une contribution de Joachim Schlör (« Ankommen in Berlin») sur le thème de l'arrivée à Berlin d'intellectuels juifs à différentes époques. Dans cet article introductif, l'auteur évoque tout d'abord la légendaire arrivée à Berlin de Moïse Mendelssohn et celle du philosophe Salomon Maïmon au dix-huitième siècle. Il est ensuite question de l'arrivé à Berlin de l'écrivain autrichien d'origine juive Karl Emil Franzos en 1887. À L'appui des Berliner Briefe de Franzos, J. Schlör analyse les réactions de l'arrivant dans une ville en pleine modernisation et tournée vers l'avenir. Citant les souvenirs de témoins de l'époque lors de leur arrivée à Berlin, l'auteur esquisse ensuite une géographie de la ville avec ses quartiers peuplés de Juifs de l'Est, et ceux où prédominent 
les Juifs de l'Ouest, davantage assimilés et intégrés, et nous donne à humer l'atmosphère de Berlin autour de 1900. Il est ensuite question du départ des Juifs de la capitale allemande après 1933 et de leur arrivée dans des villes comme New York ou Tel-Aviv et enfin de l'arrivée d'Israéliens à Berlin au début du vingt-et-unième siècle.

La première partie, consacrée aux topographies de la grande ville, s'ouvre sur l'article de Tobias Metzler («Führer durch den Text der Stadt »), qui à l'appui de guides et annuaires, destinés aux Juifs berlinois, édités dans les années 1920 et 1930 tâche de mettre en avant les concepts de ville-textes (Stadttexte) et de texte-villes (Textstädte). L'auteur y revient sur le thème de la communauté, alors en vogue en Allemagne, et sur le pessimisme culturel largement relayés par des penseurs juifs. Les guides évoqués auraient émergé de la volonté de ne pas voir disparaître l'élément juif dans une ville désormais immense et en constante mutation. Les auteurs de ces guides auraient voulu, loin de tout isolationnisme, mettre en avant l'aventure réussie d'une « symbiose judéo-allemande ». Mentionnant l'expression d'Ernst Simon «Aufbau im Untergang ", l'auteur évoque également la cartographie du Berlin juif dans les premières années du national-socialisme, parlant d'une « construction cartographique de la réalité » où l'idéologie joue un rôle important, et soulignant l'illusion de nombreux Juifs de l'époque qui n'auraient su renoncer au mythe de la fameuse «symbiose ».

Vient ensuite un article de Simone Ladwig-Winters (« Wertheim und der Glanz vom Leipziger Platz ») consacré à l'histoire berlinoise du grand magasin Wertheim et de ses propriétaires d'origine juive, de ses débuts jusqu'à sa disparition en 1945. L'auteur, qui a écrit une thèse et publié plusieurs ouvrages sur le sujet, replonge tout d'abord son lecteur (y compris à l'aide d'illustrations) dans l'ambiance du grand magasin situé sur la Leipziger Platz en 1925. Puis elle retrace brièvement l'histoire des débuts de l'enseigne en province avant que le succès ne conduise ses propriétaires jusqu'à Berlin en 1885. L'auteur nous fait part du rapport distant des Wertheim avec la religion juive, ce qui n'a pas empêché qu'ils soient victimes d'antisémitisme dès les premières années du vingtième siècle. L'histoire de la famille est brièvement retracée, ainsi que celle du bâtiment de la Leipziger Platz. S. Ladwig-Winters évoque les succès et les déboires de l'entreprise lors de la Première Guerre Mondiale et dans les années 1920, avant d'en venir à la période du nationalsocialisme. Les magasins Wertheim sont alors confrontés à une politique de boycott orchestrée par le régime. Georg Wertheim, converti depuis longtemps au christianisme, est de nouveau renvoyé à son identité juive et victime de la politique d'« aryanisation ». Après la mort de ce dernier en 1939 et la nationalisation des entrepôts situés en zone d'occupation soviétique après la guerre, l'entreprise est condamnée à disparaître. 
Sonia Goldblum, auteur d'une thèse sur la correspondance de Franz Rosenzweig, s’intéresse quant à elle dans son article («Jüdisches Forschen oder jüdisches Lernen ») à deux centres d'études juives dans le Berlin des années 1920. L'auteur resitue le développement d'institutions juives d'enseignement pour adultes dans le contexte de la « renaissance juive » en Allemagne et dans le mouvement des universités populaires (Volkshochschulbewegung). À l'appui de l'écrit programmatique de Franz Rosenzweig «Zeit ist's », S. Goldblum présente la vocation de l'Académie pour la science du judaïsme (Akademie für die Wissenschaft des Judentums), située à Berlin, à savoir de faire contrepoids à l'assimilation en rendant les catégories religieuses du judaïsme et ses textes fondamentaux de nouveau familiers aux Juifs allemands. Ce faisant, l'auteur insiste sur la volonté des instigateurs du mouvement de concilier un ancrage dans la tradition juive avec le fait de prêter allégeance à la « modernité », notamment en recourant aux méthodes de la recherche scientifique moderne. Ce sur quoi on ne saurait la contredire, si ce n'est que S. Goldblum tend semble-t-il à passer sous silence le sentiment quelque peu «anti-moderne » de la génération de Rosenzweig, par opposition à celle de Hermann Cohen. Sont ensuite évoquées différentes réalisations de l'académie ainsi que les critiques auxquelles elle fut confrontée et il est enfin question de l'histoire de la Berliner Freie Jüdische Volkshochschule. Celle-ci, davantage tournée vers la recherche que l'enseignement, a pâti de la comparaison avec l'institution francfortoise dirigée par Rosenzweig et ses méthodes d'enseignement révolutionnaires. L'auteur souligne cependant l'originalité des contenus d'enseignement de l'institution berlinoise, qui intégrait par exemple l'économie politique, la sociologie ou la psychologie.

L'article d'Eszter Gantner («Jüdische Räume in Berlin») se tourne davantage vers l'actualité puisqu'il traite des mutations de l'« espace juif » à Berlin suite à deux événements : un tournant dans la culture mémorielle et la présence croissante d'Israéliens dans la capitale allemande. Ce tournant dans la culture mémorielle serait la conséquence d'une multiplication des formes de la mémoire et d'une institutionnalisation de la mémoire ainsi que d'un changement de génération: les jeunes générations n'ont plus accès - ou, serait-il plus exact, n'auront bientôt plus accès - aux témoignages oraux des survivants de la Shoah, et sont par ailleurs caractérisées par la multiculturalité. De façon intéressante, E. Gantner détaille cette mutation de l'« espace juif » berlinois en parlant d'un passage « du souvenir à l'exotisme » défini par la présence croissante d'une culture israélienne présentée comme exotique, à côté de la culture du souvenir véhiculée par les cimetières, bains et synagogues d'autrefois. Enfin, l'auteur insiste sur le fait que cette nouvelle culture exotique existe conjointement à celle du souvenir, sans venir la remplacer - comme l'atteste la réouverture d'une école juive pour 
filles -, avant de conclure sur l'évocation d'une certaine perte de « l'honneur et de la dignité » de l'ancienne culture juive.

La seconde partie de l'ouvrage, consacrée à la vie intellectuelle et à la musique à Berlin aux dix neuvième et vingtième siècles commence avec un article de Robert Krause qui s'intéresse aux rapports de Heinrich Heine avec les Juifs berlinois ("Weil ich es immer versäumt habe, dem neuen mosaischen Gottesdienst einmal beyzuwohnen »). Partant d'une lettre de Heinrich Heine où celui-ci mentionne successivement la nouvelle bourse de Berlin et l'entreprise récente de conversion des Juifs au christianisme, l'auteur esquisse un rapprochement entre les successeurs de la Haskala à Berlin et le monde de la finance au début du dixneuvième siècle. Après avoir évoqué les rapports de Heine aux mouvements de réforme du judaïsme, $R$. Krause retrace l'histoire de la société de conversion des Juifs (Bekehrungsgesellschaft) à partir de la présentation qu'en ont donné Heinrich Heine et Karl Varnhagen von Ense. Comme le montre l'auteur, Heine et Varnhagen insistent sur le peu de succès de la mission de conversion pourtant soutenue par les souverains prussiens et tournent l'entreprise en dérision. Enfin, R. Krause revient sur l'ouvrage de Hannaah Arendt consacré à Rahel Varnhagen, soulignant l'opposition mise en lumière par la philosophe entre le «non » de Rahel Varnhagen et le « oui » de Heinrich Heine au judaïsme.

Laure Gauthier s'intéresse, quant à elle, dans son article « Arnold Schönbergs Aufenthalte in Berlin oder die Erfahrung des intensiven Auslands » aux différents séjours berlinois du compositeur et à leur influence sur sa pensée et son œuvre. On ne donnera ici qu'un bref aperçu du long et passionnant article: confronté à une critique hostile et à l'antisémitisme à Vienne, Schönberg cherche à Berlin un espace de liberté pour sa composition. Converti au christianisme en 1898, il se rapproche du judaïsme dans les années 1920 et s'investit dans la cause sioniste. C'est à Berlin qu'il rédige ses premiers articles pour la revue sioniste Pro Zion! En 1925, Schönberg est nommé à la Professur für Komposition de la Berliner Akademie et rédige alors de nombreux articles où il présente sa conception de la musique moderne. Son intérêt croissant pour le judaïsme se reflète dans le livret de son dernier opéra « Moïse et Aaron ». En 1933, il doit quitter l'Allemagne et se convertit au judaïsme à Paris. Enthousiasmé par la création de l'Etat d'Israël, c'est pourtant aux Etats-Unis qu'il finit ses jours. Berlin sera resté pour le compositeur un lieu de l'entre-deux, entre sa patrie, Vienne, et son pays d'adoption, l'Amérique.

Larticle de Monika Richarz «Eine weibliche Avantgarde » poursuit l'investigation des rapports entre Berlin et les Juifs à partir du cas des femmes juives dans les universités berlinoises et des femmes diplômées de ces mêmes universités de la fin du dix-neuvième siècle à 1933. L'auteur part du constat que c'est davantage la réticence de l'Univer- 
sité allemande à accueillir des femmes que l'antisémitisme qui a limité l'accès de femmes juives à l'Université et souligne que ces dernières furent généralement plus nombreuses à franchir cet obstacle et qu'elles le firent en moyenne plus tôt que les femmes non-juives. M. Richarz rappelle l'éducation qui était réservée aux femmes en Allemagne avant que ne soit autorisée leur inscription à l'Université. Puis, elle met en avant et tâche d'expliquer la surreprésentation d'étudiantes juives, en particulier venues de l'Empire russe, dès que l'inscription de femmes à l'Université a été rendu possible. La vie des étudiantes juives dans les universités allemandes est ensuite évoquée, ainsi que les matières qui leur étaient réservées et l'attitude des Professeurs à leur encontre. Puis, l'auteur se livre à un long et quelque peu fastidieux inventaire des différents métiers exercés par des femmes juives diplômées avant 1933 et cite quelques exemples de brillantes réussites dans les domaines des sciences, du journalisme ou du droit, parmi lesquelles certaines biographies retiennent néanmoins l'intérêt du lecteur.

Enfin, l'ouvrage se termine sur l'article de Céline Trautmann-Waller («Jüdisches und Humboldtianisches Berlin») consacré aux rapports des frères Humboldt aux milieux juifs berlinois. L'auteur souligne d'emblée l'importance d'intellectuels juifs dans la formation des frères Humboldt, tout en restant prudente face au « myhte » du dialogue judéoallemand qui se serait développé dans les salons berlinois. Les amitiés entre les frères Humboldt et des figures comme Moïse Mendelssohn ou Henriette Herz retiennent tout d'abord l'attention, ainsi que leur engagement pour l'émancipation des Juifs. Puis C. Trautmann-Waller consacre plusieurs pages aux Juifs ayant rédigé des biographies d'Alexander von Humboldt dans un but de promotion et de popularisation des sciences naturelles. Plusieurs exemples viennent illustrer le fait que ces ouvrages ont souvent cherché, sous l'influence d'une croyance immodérée en la marche du progrès scientifique et social, à transformer l'explorateur et savant en héros. L'esprit cosmopolite de Humboldt aurait si on en croit l'auteur, attiré à lui la sympathie des Juifs allemands à une époque (après 1871) où le nationalisme galopant leur était défavorable. Enfin, C. Trautmann-Waller met en avant le rapprochement entre des idées libérales de gauche, un intérêt pour les sciences naturelles et la célébration des frères Humboldt que l'on observe dans les différentes sociétés Humboldt (Humboldt-Vereine) au dix neuvième et au début du vingtième siècle.

En somme, l'ouvrage traite, de façon très érudite et relativement cohérente, différents aspects des « relations » qu'ont pu entretenir les Juifs avec la ville de Berlin aux dix-neuvième et vingtième siècle autour d'un pôle «topographique » et d'un pôle culturel. Le recueil intéressera les historiens du monde juif allemand aussi bien que les «berlinolo- 
gues » ou les spécialistes d'histoire culturelle intéressés par les différentes méthodes d'investigation mises en œuvre par les auteurs.

François Prolongeau

Paul Nizon : Parisiana, Matthes \& Seitz Berlin, 2015, 149 Seiten.

"On monte à Paris": In Frankreich weiß jeder, dass der Ausdruck nicht geographisch zu vestehen ist, sondern etwas mit Aufstieg zu tun hat. Er zeigt, wie die französische Hauptstadt bis heute für viele Kunstschaffende der Ort ist, wo sie endlich erfahren können, ob sie auch dazu gehören, ob sie echte Künstler sind. Aus diesem Grund wurde sie auch vielen Malern, Schriftstellern, Musikern zur Wahlheimat. So gilt es auch für Paul Nizon: Unter dem Titel Parisiana erscheint vom aus Bern stammenden, seit fast vierzig Jahren in Paris lebenden Schweizer Schriftsteller eine Textsammlung, die verschiedene, zum Teil schon bekannte Texte mit Parisbezug aus dem Gesamtwerk enthält.

In Leben und Werk Nizons haben Metropolen auch immer einen ganz speziellen Platz eingenommen: Bevor Nizon sich Mitte der siebziger Jahre fürs Pariser Exil entschied, hatte er schon mehrere solcher Erfahrungen in Rom, Barcelona oder auch London gemacht. Doch es handelte sich damals nur um Arbeitsaufenthalte. Den eigentlichen Bruch mit dem damaligen, 'komfortablen' Leben, das er als angesehener Kunstkritiker und Schriftsteller in Zürich führte - der 1975 erschienene Roman Stolz hatte ihm viel Anerkennung im deutschsprachigen Raum gebracht -, vollzog er erst durch die Selbstverpflanzung nach Paris. Mit anderen Worten kehrte er dem früheren Leben radikal den Rücken $\mathrm{zu}$, indem er auf jeden sozialen Status verzichtete. Im Gespräch mit Martin Simons, das im Anhang steht, bekennt Nizon: "Ich denke, ich wäre in der Schweiz nach 'Stolz', dessen Protagonist nicht von ungefähr ein Lebensverweigerer ist, nicht weitergekommen. Meine stofflichen Reserven waren erschöpft, und für die Selbsterneuerung fehlte die Herausforderung." Die Erneuerung des Schriftstellers ging also notwendigerweise übers Pariser Exil.

In Paris kann sich Nizon endlich der Poetenexistenz aussetzen, die er als einzigmögliche Antwort nicht nur auf die Heimatlosigkeit, sondern auch auf die Themenlosigkeit betrachtet: "Ich fahre zur Zeit zwecklos viel, als könnte ich so das Schreiben in Gang bringen." Ferner heißt es: "Ich rase ja neuerdings wieder kreuz und quer durch die Stadt, fresse mich richtig voll mit Pariser Bildern, Lebensmaterial (...) In-GangSetzung der inneren Schreibmaschine im Sinne der Übersättigung, die das respondierende Formulieren wachruft." Hier nehmen die von Nizon genannten "Ateliers" einen ganz besonderen Platz ein: Auf dem Weg zu diesen gemieteten Buden, Mansarden, Kleinwohnungen, in die sich der Schriftsteller seit Jahren zum Schreiben zurückzieht, vollzieht 
sich die Verwandlung der zivilen Person ins Ich seiner Fiktionen, die eigentlich Autofiktionen sind. Wichtig sind also auch die Bus- bzw. U-Bahn-Fahrten. Auch sie gehören zum Schaffensprozess.

Paul Nizon ist zum Pariser Schriftsteller geworden: In dieser Stadt wie in keiner anderen ist er fest literarisch beheimatet. Ihr verdankt er "die Verschmelzung von Stadt-, Künstler- und Existenzroman", die seinen literarischen Stoff ausmacht. Aus diesem Grund lassen sich seine Bücher auch wie Huldigungen an die französische Hauptstadt lesen, nicht zuletzt Das Jahr der Liebe (1981), insofern Nizons schönstes Buch, als es die Wechselwirkung von Leben und Schreiben in einer seltenen melancholisch-kreativen Stimmung dem Leser zu kommunizieren vermag. Der letzte Teil von Parisiana besteht logischerweise aus langen Auszügen aus dem Jahr der Liebe, dem Buch par excellence, in dem das schreibende Ich selber um den Roman kämpft, an dessen Schreibprozess der Leser auch gleichzeitig teilnehmen kann. In einer Mischung von Urbomanie und Erotomanie schafft es das Ich, eine sprachliche Antwort auf die immer wiederkehrende Frage "Wo ist das Leben?" (unter diesem Titel erschien 1983 ein erstes Lesebuch Nizons) $\mathrm{zu}$ finden. In besonders gelungenen Straßenszenen erweist sich Nizon als treuer Erbe der "flâneurs", wie sie mit Baudelaire und Benjamin in die Literaturgeschichte eingegangen sind. Der flâneur Nizon fühlt sich unter der Menge zu Hause und praktiziert, an Michel de Certeau unbewusst anlehnend, indem er durch die Stadt wandert, deren Sprache.

Gegen Ende der Textsammlung wird der Leser die ästhetisch seltene Erfahrung eines langen, auch dem Jahr der Liebe entnommenen Satzes machen können, der sich über circa fünfzehn Seiten erstreckt und der Beschreibung einer Busfahrt entspricht. Während dieser Fahrt öffnet das Ich all seine Sinne der ihm umliegenden Stadt, es wird dabei beinahe anonym und kommt zu dem Schluss: "Ich kann dich nicht sagen, doch ich kann dich fahren". Durch die sich hier vollziehende Machtergreifung der Sprache erreicht die Schreibtätigkeit hier wohl ihren Höhepunkt. Die Wirklichkeit kommt in der Sprache zustande und der Leser bekommt vielleicht, nachdem er das Buch geschlossen hat, zum ersten Mal den Eindruck, dass ihm das Leben in seiner ganzen narrativen Dimension kommuniziert wurde. 
